This article is licensed under the Creative Commons Attribution-NonCommercial 4.0 International License (CC BY-NC) (http://www.karger.com/Services/OpenAccessLicense). Usage and distribution for commercial purposes requires written permission.

\title{
Endocytoscopic Observation of Non-Ampullary Mucosal Duodenal Cancer
}

\author{
Youichi Kumagai $^{\mathrm{a}}$ Morihiro Higashi $^{\mathrm{b}}$ Shunsuke Muramatsu ${ }^{\mathrm{a}}$ \\ Erito Mochiki ${ }^{a}$ Hideyuki Ishida ${ }^{a}$ \\ ${ }^{a}$ Department of Digestive Tract and General Surgery, Saitama Medical Center, Saitama \\ Medical University, Saitama, Japan; ${ }^{b}$ Department of Pathology, Saitama Medical Center, \\ Saitama Medical University, Saitama, Japan
}

\section{Keywords}

Endocytoscopy system · Non-ampullary duodenal epithelial tumor - Duodenal cancer .

Mucin phenotype

\begin{abstract}
Our previous study of duodenal adenoma using an endocytoscopy system (ECS) demonstrated that disappearance of goblet cells and spindle-shaped nuclei with loss of polarity were characteristic features. In addition, round duct openings and finger-like projections were observed in tubular adenoma and villous adenoma, respectively. Here, we retrospectively investigated six cases of histologically proven sporadic non-ampullary mucosal duodenal cancer (NAMDC) using ECS. Immunohistochemistry for CD10, MUC2, MUC5AC, and MUC6 was employed to determine the mucin phenotype in addition to conventional HE histology. Immunohistochemistry revealed one case involving the duodenal bulb that was considered to be the mixed type. The other five cases, located in the second or third portion, were considered to be the intestinal type. Vital staining of the mixed-type case was considered insufficient for ECS observation because of surface mucus. However, all five cases of intestinal-type duodenal cancer
\end{abstract}


demonstrated a villous structure, disappearance of goblet cells and enlarged nuclei with loss of polarity. Tubular structures were admixed in four of those cases. Four cases demonstrated oval-shaped nuclei, and one case had spindle-shaped nuclei. Cases showing spindle-shaped nuclei in most of the lesion were diagnosed histologically as cancer in adenoma where the adenomatous component of the tumor was dominant. Oval-shaped nuclei and nuclear enlargement are the characteristic features of NAMDC revealed by ECS and are included among the histological criteria used for diagnosis. ECS offers the potential to perform real-time histological diagnosis of NAMDC in vivo.

(C) 2020 The Author(s) Published by S. Karger AG, Basel

\section{Introduction}

Epithelial duodenal tumors are rare, and the incidence of duodenal adenoma revealed by upper GI endoscopy examination is reported to be less than $0.4 \%$ [1]. On the other hand, the widespread application of upper GI endoscopy in the last 2 or 3 decades has led to an increase in the number of duodenal cancers being discovered [2].

Several studies have reported the usefulness of magnifying endoscopic observation with narrow-band imaging (NBI-ME) for evaluation of non-ampullary duodenal epithelial tumor (NADET) [3]. However, in those studies, it was not possible to distinguish non-ampullary mucosal duodenal cancer (NAMDC) from duodenal adenoma because NBI-ME does not allow direct observation of surface cell morphology.

The endocytoscopy system (ECS) is a novel ultra-high-magnification endoscope that allows observation at the cellular level [4]. We have previously reported the ECS features of normal duodenal mucosa and duodenal lesions in patients with familial adenomatous polyposis [5]. In the normal duodenal mucosa, nuclei were observed to have a regular arrangement in each villus with sparse goblet cells, whereas adenomatous lesions showed disappearance of goblet cells and spindle-shaped nuclei with loss of polarity. In addition, round duct openings and finger-like projections were observed in tubular adenoma and villous adenoma, respectively. With regard to duodenal cancer, several articles have reported the ECS features of NAMDC $[6,7]$. However, as those studies recruited only a small number of NAMDC cases, detailed comparison between the ECS features and conventional histology of NAMDC is still insufficient. Here, we evaluated the ECS features of NAMDC with reference to conventional histology combined with the mucin phenotype.

\section{Case Series}

We retrospectively investigated six cases of histologically proven NAMDC observed by ECS at the Saitama Medical Center, Saitama Medical University, between 2015 and 2019. 


\section{Case Reports in Gastroenterology}

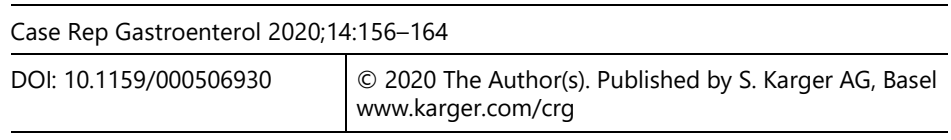

Kumagai et al.: Endocytoscopic Observation of Non-Ampullary Mucosal Duodena Cancer

\section{Equipment}

We employed the GIF-H290EC for this study. This ECS has one lens whose magnification can be increased consecutively from the conventional endoscopy level through to the NBI-ME level (approximately $\times 100$ ), and to a maximum of $\times 500$ (tissue field of view, $570 \times 500 \mu \mathrm{m}$ ) using a hand lever.

\section{In vivo Observation}

Prior to ECS observation, we observed the lesions using white light and NBI with or without slight magnification (approximately $\times 100$, conventional NBI-ME level). We then sprayed the lesion with $0.5-1 \mathrm{~mL}$ of $2 \%$ methylene blue. Approximately $1 \mathrm{~min}$ after spraying the dye on the target mucosa, we brought the ECS lens in contact with the lesion and finally zoomed up to the maximum magnification. In cases of insufficient vital staining, re-staining was performed.

\section{Histological Examination}

All six lesions were surgically resected. In addition to conventional HE staining, we performed immunohistochemical analysis for CD10 (CD10 PA0270), Muc-2 (Muc-2 Glycoprotein PA0155), MUC-5AC (Muc-5AC Glycoprotein PA0052), and MUC-6 (Muc-6 Glycoprotein PA0053) (all from Leica Biosystems Newcastle Ltd., UK) for determination of the mucin phenotype. The intestinal type was defined as a phenotype positive for only intestinal markers (MUC2 and/or CD10). Similarly, the gastric type was defined as a phenotype positive for only gastric markers (MUC5AC and/or MUC6). Tumors positive for both the gastric and intestinal phenotypes were considered to be of the mixed type.

\section{Methods}

From the ECS images, we evaluated the presence or absence of goblet cells, tubular structures or villous structures, loss of nuclear arrangement polarity, nuclear enlargement, and nuclear shape. We then compared the ECS images with histological sections that matched the region observed by ECS.

\section{Results}

Detailed data for all six lesions are shown in Table 1. Macroscopically, all cases were of the elevated type with a median diameter of $48.5 \mathrm{~mm}$ (range 25-70 $\mathrm{mm}$ ). Immunohistochemical analysis suggested that one case involving the duodenal bulb was the mixed type, whereas the other five cases were the intestinal type and located in the 2nd or 3rd portion of the duodenum. Vital staining of the case considered to be the mixed type (case 1) was insufficient for 


\section{Case Reports in Gastroenterology} \begin{tabular}{l|l}
\hline Case Rep Gastroenterol 2020;14:156-164 \\
\hline DOI: 10.1159/000506930 & $\begin{array}{l}\text { @ 2020 The Author(s). Published by S. Karger AG, Basel } \\
\text { www.karger.com/crg }\end{array}$ \\
\hline
\end{tabular}

Kumagai et al.: Endocytoscopic Observation of Non-Ampullary Mucosal Duodenal Cancer

ECS observation because of surface mucus. In all five cases of intestinal-type duodenal cancer, ECS demonstrated a villous structure, whereas tubular structures are admixed in four of these cases. In all five cases, disappearance of goblet cells and loss of polarity were observed, and the cancerous area showed enlarged nuclei. Four cases were found to have oval-shaped nuclei, and one case had spindle-shaped nuclei. Loss of histological structure and nuclear morphology demonstrated by ECS were matched to histological sections in most parts of the tumor (Fig. 1). Among them, cases 2 and 3 showed spindle-shaped nuclei in most of the lesion. Case 2 showed only spindle-shaped nuclei, whereas case 3 showed enlarged oval-shaped nuclei in a limited area among the villous adenomatous component (Fig. 2). These cases were diagnosed histologically as cancer in adenoma where the adenomatous component was dominant in the tumor.

\section{Discussion}

The ECS technique, which allows endoscopic observations to be conducted at the cellular level, was first developed by our own group and applied for clinical use in 2003, when we reported in vivo observation of a case of esophageal cancer [8]. The GIF-H290EC was produced as an experimental prototype in 2015 , and finally released onto the market in 2018 [4]. ECS allows detailed diagnosis of the gastrointestinal tract at the cellular level but has now been applied to all parts of the digestive tract and other structures $[9,10]$. The most notable use of ECS is for real-time, high-resolution diagnosis of cellular abnormalities.

In the context of NADETs, ECS observation has two apparent diagnostic advantages. First, real-time histological diagnosis using ECS may allow omission of biopsy histology. Tissue sampling from NADETs using endoscopic forceps biopsy sometimes induces severe fibrosis, creating difficulty with subsequent endoscopic treatment [11]. For this reason, omission of biopsy histology would be of help to endoscopists. Second, it has been reported that the accuracy of duodenal biopsy sampling is relatively low (71.6\%) [12]. Because duodenal cancer arises from duodenal adenoma (through the adenoma-carcinoma sequence), any cancerous region in the tumor is limited. Therefore, it is difficult for endoscopists to obtain adequate biopsy samples from the cancerous area. The use of ECS may allow more accurate endoscopic diagnosis or more adequate targeted biopsy for NADETs.

No pathological criteria for diagnosis of NADETs have yet been established. Recently, it has been reported that NADETs include two main categories based on the mucin phenotype: intestinal or gastric [13]. The main pattern of duodenal carcinogenesis is considered to be the "adenoma-carcinoma sequence," similar to colorectal tumors. Thus, the main phenotype of NADETs might be the intestinal type. Gastric-type NADETs coexist with gastric foveolar metaplasia, duodenal gastric heterotopia, and/or Brunner's gland hyperplasia. NADETs of the gastric type tend to be located in the proximal duodenum and have higher malignant potential than those of the intestinal type [3]. Five of the six cases in our present series were considered to be of the intestinal type that were clearly stained and observed using ECS. On the other hand, one case considered to be of the mixed type could not be stained adequately because of surface mucus. ECS observation of the stomach is sometimes difficult because surface mucus prevents vital staining. Gastric or mixed-type NADETs may also secrete rich mucus that hinders vital staining. 
In general, histological diagnosis of mucosal duodenal cancer is made on the basis of features such as irregular nuclear size and shape, an increased nuclear:cytoplasmic (N/C) ratio, loss of polarity in nuclear arrangement, and structural abnormality [14]. In our present series, all cases of NAMDC showed a villous structure, disappearance of goblet cells, and loss of polarity. However, these features were also observed in duodenal adenoma. In our series, the most apparent features of NAMDC revealed by ECS in comparison with duodenal adenoma were oval-shaped nuclei and nuclear enlargement. An oval or round nuclear shape is a histological feature of duodenal cancer (whereas duodenal adenoma has spindle-shaped nuclei). Further, nuclear enlargement demonstrated by ECS may reflect an increased N/C ratio. These findings can help to target subsequent biopsy to an adequate cancerous area or allow biopsy histology to be omitted. However, the present study was retrospective, conducted at a single center, and included only a limited number of cases. Therefore, accumulation of further NADET cases and more detailed comparison with histopathology are mandatory. In addition, ECS itself is an intrinsic limitation in that it provides information only on surface epithelial cells because of the character of vital staining. If cancerous cells are located on the subsurface of the epithelium only, they would not be recognized. This is the fundamental limitation of ECS observation. However, in our present series, the fact that ECS observation of NAMDC was able to clearly visualize the histological character of the cancerous area was encouraging.

In conclusion, ECS observation of NAMDC has the potential to allow real-time histological diagnosis in vivo, as oval-shaped nuclei and nuclear enlargement are the characteristic features of this cancer. Because ECS can clearly demonstrate the cell arrangement and nuclear morphology of NADETs, we believe it can provide sufficient information to suggest whether more detailed endoscopic evaluation would be justified.

\section{Acknowledgement}

This study was supported by MEXT KAKENHI Grant number 19 K08477.

\section{Statement of Ethics}

Written informed consent was obtained from all patients, and this study was approved by the hospital ethics committee.

\section{Disclosure Statement}

Author Y.K. borrowed a GIF-H290EC from Olympus Medical Systems Co. without payment.

\section{Funding Sources}

This study was supported by MEXT KAKENHI Grant number 19K08477. 


\section{Case Reports in Gastroenterology}

\section{Author Contributions}

Y.K. was involved in the study concept and design, analysis and interpretation of the data and drafting of the manuscript. H.I., E.M., and S.M. were involved in accumulation of the data and critical revision of the manuscript for important intellectual content. M.H. was involved in pathological analysis.

\section{References}

1 Jepsen JM, Persson M, Jakobsen NO, Christiansen T, Skoubo-Kristensen E, Funch-Jensen P, et al. Prospective study of prevalence and endoscopic and histopathologic characteristics of duodenal polyps in patients submitted to upper endoscopy. Scand J Gastroenterol. 1994 Jun;29(6):483-7.

2 Goda K, Kikuchi D, Yamamoto Y, Takimoto K, Kakushima N, Morita Y, et al. Endoscopic diagnosis of superficial non-ampullary duodenal epithelial tumors in Japan: multicenter case series. Dig Endosc. 2014 Apr;26 Suppl 2:23-9.

3 Toya Y, Endo M, Akasaka R, Urushikubo J, Gonai T, Asakura K, et al. Clinicopathological features and magnifying chromoendoscopic findings of non-ampullary duodenal epithelial tumors. Digestion. 2018;97(3):219-27.

4 Kumagai Y, Takubo K, Kawada K, Higashi M, Ishiguro T, Sobajima J, et al. A newly developed continuous zoom-focus endocytoscope. Endoscopy. 2017 Feb;49(2):176-80.

5 Kumagai Y, Watanabe Y, Ishida H. Endocytoscopic observation of duodenal polyps associated with familial adenomatous polyposis: report of four cases. Dig Endosc. 2015 Nov;27(7):778.

6 Neumann H, Kudo S, Vieth M, Neurath MF. Real-time in vivo histologic examination using a probe-based endocytoscopy system for differentiating duodenal polyps. Endoscopy. 2013;45 Suppl 2 UCTN:E53-4.

7 Miyamoto S, Kudo T, Abiko S, Ono S, Shimizu Y, Matsuno Y, et al. Endocytoscopy of superficial nonampullary duodenal epithelial tumor: two cases of tubular adenocarcinoma and adenoma. Am J Gastroenterol. 2017 Nov;112(11):1638.

8 Kumagai Y, Monma K, Kawada K. Magnifying chromoendoscopy of the esophagus: in-vivo pathological diagnosis using an endocytoscopy system. Endoscopy. 2004 Jul;36(7):590-4.

9 Newton RC, Kemp SV, Shah PL, Elson D, Darzi A, Shibuya K, et al. Progress toward optical biopsy: bringing the microscope to the patient. Lung. 2011 Apr;189(2):111-9.

10 Ohigashi T, Kozakai N, Mizuno R, Miyajima A, Murai M. Endocytoscopy: novel endoscopic imaging technology for in-situ observation of bladder cancer cells. J Endourol. 2006 Sep;20(9):698-701.

11 Kakushima N, Ono H, Takao T, Kanemoto H, Sasaki K. Method and timing of resection of superficial nonampullary duodenal epithelial tumors. Dig Endosc. 2014 Apr;26 Suppl 2:35-40.

12 Kinoshita S, Nishizawa T, Ochiai Y, Uraoka T, Akimoto T, Fujimoto A, et al. Accuracy of biopsy for the preoperative diagnosis of superficial nonampullary duodenal adenocarcinoma. Gastrointest Endosc. 2017 Aug;86(2):329-32.

13 Mitsuishi T, Hamatani S, Hirooka S, Fukasawa N, Aizawa D, Hara Y, et al. Clinicopathological characteristics of duodenal epithelial neoplasms: focus on tumors with a gastric mucin phenotype (pyloric gland-type tumors). PLoS One. 2017 Apr;12(4):e0174985.

14 Yoshida M, Shimoda T, Abe M, Kakushima N, Kawata N, Takizawa K, et al. Clinicopathological characteristics of non-ampullary duodenal tumors and their phenotypic classification. Pathol Int. 2019 Jul;69(7):398-406. 


\section{Case Reports in Gastroenterology}
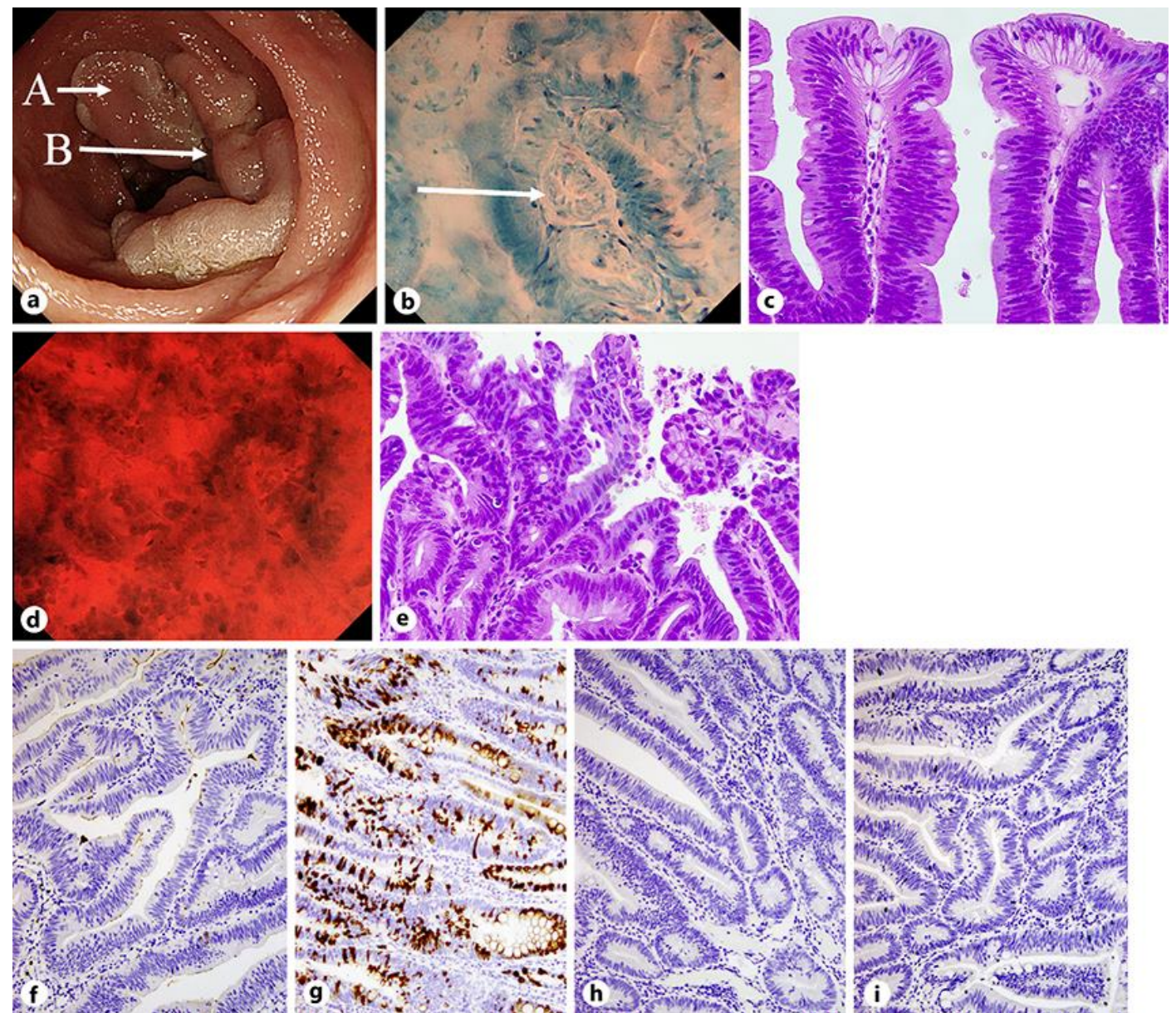

Fig. 1. a Non-magnified view of intestinal mucosal duodenal cancer (case 5). This cancer was whitish (partially reddish), flat, and elevated. Endocytoscopic pictures in $\mathbf{b}$ and $\mathbf{d}$ were obtained from region $\mathrm{A}$ and region $B$, respectively. $\mathbf{b}$ In region A, duct openings (tubular structure: arrow) inside areas of villus formation with spindle-shaped and enlarged nuclei are evident. $\mathbf{c}$ Histologically, villus structures with spindle-shaped nuclei are also evident, suggesting this area was the adenomatous component $(\times 400)$. $d$ In lesion B, enlarged oval-shaped nuclei with marked destruction of tubular or villous structures are evident. e Histologically, this lesion was diagnosed as duodenal cancer showing round and oval enlarged nuclei with loss of nuclear polarity and marked destruction of tubular or villous structures $(\times 400)$. $f-i$ This tumor was considered to be intestinal type from the immunohistochemical analysis $(\times 200) . \mathbf{f} \mathrm{CD} 10$ negative. $\mathbf{g}$ MUC2 positive. $h$ MUC5AC negative. i MUC6 negative. 


\section{Case Reports in Gastroenterology}

Case Rep Gastroenterol 2020;14:156-164
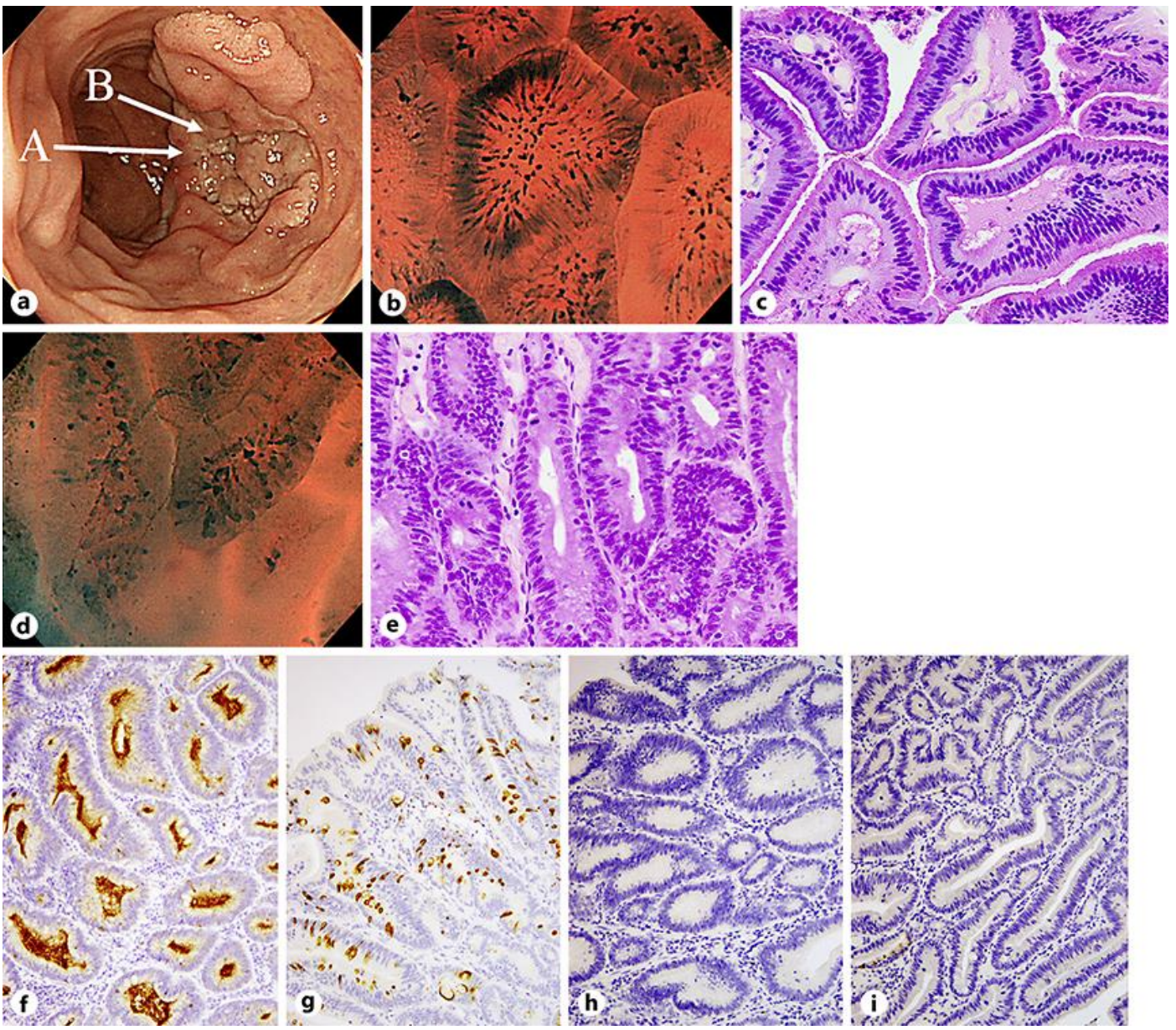

Fig. 2. a Non-magnified view of an intestinal mucosal duodenal cancer (case 3). Endocytoscopic pictures in $\mathbf{b}$ and $\mathbf{d}$ were obtained from region $A$ and region B, respectively. $\mathbf{b}$ Region A shows a villous structure with spindle-shaped nuclei. Endocytoscopically, most of the mucosal surface of this tumor appeared similar to this picture. c Histologically, villous structures with spindle-shaped nuclei are evident. The villous adenomatous component is dominant in this tumor $(\times 400)$. d In lesion B (limited area), enlarged ovalshaped nuclei with villous structures are evident. e Histologically, this region was diagnosed as duodenal cancer showing enlarged round and oval nuclei with loss of nuclear polarity with tubular or villous structures $(\times 400) . \mathbf{f}-\mathbf{i}$ This tumor was considered to be intestinal type from the immunohistochemical analysis $(\times 200)$. $\mathbf{f}$ CD10 positive. $\mathbf{g}$ MUC2 positive. $\mathbf{h}$ MUC5AC negative. $\mathbf{i}$ MUC6 negative. 
 Gastroenterology}

\begin{tabular}{l|l}
\hline Case Rep Gastroenterol 2020;14:156-164 \\
\hline DOI: 10.1159/000506930 & $\begin{array}{l}\text { (c) 2020 The Author(s). Published by S. Karger AG, Basel } \\
\text { www.karger.com/crg }\end{array}$ \\
\hline
\end{tabular}

Kumagai et al.: Endocytoscopic Observation of Non-Ampullary Mucosal Duodenal Cancer

Table 1. Clinical and endocytoscopic characteristics of six cases

\begin{tabular}{|c|c|c|c|c|c|c|c|c|c|c|c|c|}
\hline Case & Gender & $\begin{array}{l}\text { Age, } \\
\text { years }\end{array}$ & Location & $\begin{array}{l}\text { Maximal } \\
\text { diameter, } \\
\text { mm }\end{array}$ & $\begin{array}{l}\text { Macroscopic } \\
\text { appearance }\end{array}$ & $\begin{array}{l}\text { Mucin } \\
\text { phenotype }\end{array}$ & $\begin{array}{l}\text { Goblet } \\
\text { cell }\end{array}$ & $\begin{array}{l}\text { Villus } \\
\text { structure }\end{array}$ & $\begin{array}{l}\text { Tube } \\
\text { structure }\end{array}$ & $\begin{array}{l}\text { Loss of } \\
\text { polarity }\end{array}$ & $\begin{array}{l}\text { Nuclear } \\
\text { shape }\end{array}$ & $\begin{array}{l}\text { Nuclear } \\
\text { enlargement }\end{array}$ \\
\hline 1 & M & 79 & bulbs & 70 & elevated & mixed & \multicolumn{6}{|c|}{ poor vital staining } \\
\hline 2 & M & 67 & 2nd portion & 47 & elevated & intestinal & - & + & + & + & spindle & + \\
\hline 3 & $\mathrm{~F}$ & 50 & 2nd portion & 50 & elevated & intestinal & - & + & + & + & oval & + \\
\hline 4 & $\mathrm{~F}$ & 68 & 2nd portion & 40 & elevated & intestinal & - & + & + & + & oval & + \\
\hline 5 & $\mathrm{~F}$ & 69 & 2nd portion & 50 & elevated & intestinal & - & + & + & + & oval & + \\
\hline 6 & $\mathrm{M}$ & 66 & 3rd portion & 25 & elevated & intestinal & - & + & - & + & oval & + \\
\hline
\end{tabular}

